
allemande

46-1 | 2014

Les fondements normatifs de l'État constitutionnel moderne en Allemagne. Une approche pluridisciplinaire

Homogénéité, révision constitutionnelle et décision fondamentale : reprise et critique de concepts schmittiens chez Böckenförde et Capitant

Jean-Claude Monod

\title{
OpenEdition
}

Journals

Édition électronique

URL : https://journals.openedition.org/allemagne/1302

DOI : 10.4000/allemagne.1302

ISSN : 2605-7913

Éditeur

Société d'études allemandes

Édition imprimée

Date de publication : 30 juin 2014

Pagination : 111-124

ISSN : 0035-0974

Référence électronique

Jean-Claude Monod, « Homogénéité, révision constitutionnelle et décision fondamentale : reprise et critique de concepts schmittiens chez Böckenförde et Capitant », Revue d'Allemagne et des pays de langue allemande [En ligne], 46-1 | 2014, mis en ligne le 29 juillet 2019, consulté le 18 mai 2021. URL : http://journals.openedition.org/allemagne/1302 ; DOI : https://doi.org/10.4000/allemagne.1302

Ce document a été généré automatiquement le 18 mai 2021

Revue d'Allemagne et des pays de langue allemande 


\title{
Homogénéité, révision
}

constitutionnelle et décision

\section{fondamentale : reprise et critique de concepts schmittiens chez Böckenförde et Capitant}

\author{
Jean-Claude Monod
}

Comment reconstruire un État démocratique, libéral, après le désastre nazi, en faisant en sorte que cet État se tienne constitutionnellement à l'écart de tout «État total », mais en faisant également en sorte qu'il ne soit pas affecté des mêmes failles que la République de Weimar? Comment construire un État qui ne soit pas une force d'oppression, mais de protection, sans être néanmoins trop faible pour assurer l'unité de la société, l'unité sociale, et par là trop faible pour s'assurer le soutien d'une large majorité de la population? Comment garantir et stabiliser les conditions de possibilité d'une démocratie libérale capable de résister aux assauts de ses ennemis - mieux capable, là encore, que ne l'a été Weimar? On comprend que de telles questions ne pouvaient manquer de se poser aux juristes et constitutionnalistes allemands après 1945. Faut-il s'étonner et s'affliger du fait que certains des plus éminents d'entre eux, comme Böckenförde, aient cherché à y répondre en faisant usage - un usage critique, sélectif et politiquement réorienté - de certaines analyses et de certains concepts venus de Carl Schmitt? C'est-à-dire, comme chacun sait, d'un juriste ou du plus fameux juriste rallié au III ${ }^{e}$ Reich, non seulement rallié mais impliqué et compromis dans certaines des législations les plus détestables de ce régime? On peut s'en étonner, bien sûr. Mais pour s'en indigner a priori, il faut croire que Carl Schmitt n'a jamais été autre chose qu'un juriste nazi - rien d'autre, et rien de plus. Certains le croient, et veulent faire partager cette conviction d'une façon quelque peu comminatoire et performative, en nous intimant de ne même plus nommer Carl Schmitt sans faire précéder son nom de la mention «le nazi (Carl Schmitt) » et/ou de ne lui accorder le titre de juriste qu'entre guillemets. Il n'y aurait rien à tirer de cette pensée, pas le moindre "grain à moudre " 
théorique, même dans les écrits antérieurs à 1933, Le Concept du politique, Théorie de la constitution, Légalité et légitimité ou Le gardien de la constitution. Ces affirmations (ou négations) doivent être placées en regard des usages juridiques et des relectures de ces mêmes textes (et d'autres) réalisés par certains des plus grands constitutionnalistes allemands, mais aussi français, au moment de leur parution et surtout après 1945. Je m'en tiendrai ici essentiellement à Böckenförde et Capitant.

Cela ne veut pas dire, bien sûr, qu'on ne puisse s'interroger sur certains silences, sur certains effets politiques, sur certaines prémisses non-questionnées, sur certaines conclusions attachées à ces usages de concepts schmittiens dans un cadre républicain ou libéral, démocratique.

3 Mais pour mener à bien sérieusement un tel examen critique, il faut se donner les moyens de comprendre pourquoi des personnages de la stature intellectuelle, morale et politique de Böckenförde ou de Capitant ont reconnu une certaine pertinence à certaines problématiques et analyses schmittiennes. Je commencerai donc par là, avec l'interprétation de la crise de Weimar par Böckenförde et Capitant. Dans cette interprétation, plusieurs thèmes schmittiens prennent une importance particulière: l'homogénéité politique ; les limites de la révision constitutionnelle ; le rapport entre la Constitution et la forme politique. J'examinerai comment Böckenförde et Capitant reprennent ces thèmes, après quoi j'aborderai la question des fondements normatifs de l'État dont la position ou la résolution implique, pour Böckenförde comme pour Capitant, de s'écarter résolument de Schmitt au plan axiologique - même si Schmitt a sans doute indiqué le lieu du problème.

\section{Homogénéité}

4 Dans un article de 1960, "Entstehung und Wandel des Rechtsstaatsbegriffs ", Böckenförde écrit : «Ce qui a conduit à [la] chute [de la République de Weimar] n'a pas été un manque de liberté dans l'ordre juridico-étatique, mais un manque d'homogénéité politique et de loyauté démocratique chez les différents groupes et dans le peuple lui-même $»^{1}$.

5 Ce diagnostic confirme partiellement celui de Schmitt dans les années 1920, mais il en transforme en profondeur les termes. Böckenförde reprend ainsi l'interrogation de Schmitt à propos de l'« homogénéité » nécessaire au fonctionnement d'une démocratie, et de la légitimité de la constitution qui est liée à la reconnaissance de l'unité politique d'où elle émane. On trouvait cette thématique dans l'essai de 1923, Die geistesgeschichtliche Lage des heutigen Parlamentarismus: qu'est-ce qui fait l'unité d'un peuple? La question du demos se pose toujours en démocratie, même si les approches de la Constitution que Schmitt qualifie de formalistes ou de normativistes évitent cette question en l'excluant de la science du droit. Il y a pourtant un fondement préjuridique de l'État, qui tient à une certaine unité politique. Pour Schmitt, cette unité politique se construit toujours, comme on sait, par différenciation d'avec un "autre", le barbaros des Grecs, l'étranger ou l'ennemi politique. C'est évidemment le danger de la pensée de Schmitt que de penser l'unité et l'homogénéité politique en en faisant ressortir l'hostilité potentielle. Et en en justifiant parfois, comme dans cet article de 1926, la dimension discriminante ou discriminatoire : « une démocratie [...] peut exclure une partie de la population sans cesser d'être une démocratie, [...] en général les démocraties jusqu'à présent ont toujours eu des esclaves ou des hommes qui, d'une 
manière ou d'une autre, ont été totalement ou partiellement privés de droit $»^{2}$. Il est vrai que Schmitt citait les démocraties anglaise et française de son temps, qui maintenaient d'immenses populations colonisées dans un état de privation partielle de droits sans que grand monde y trouve à redire - surtout pas Schmitt, qui enfonce le clou dans un sens xénophobe : « la force politique d'une démocratie se manifeste à sa capacité d'écarter ou de tenir éloigné l'étranger ", " l'égalité de tous les hommes en tant qu'hommes n'est pas de la démocratie mais une certaine forme de libéralisme $»^{4}$.

6 Après la Seconde Guerre mondiale et l'expérience nazie, Böckenförde ne reprend évidemment pas cette orientation-là de la réflexion de Schmitt sur l'homogénéité : il reformule celle-ci en termes d'homogénéité politique et sociale, c'est-à-dire d'unité du peuple et de loyauté à l'égard de l'État, et en fonction des facteurs de la reconnaissance de sa légitimité.

7 Il s'appuie donc plutôt sur les chapitres de la Théorie de la constitution de 1928 consacrés à la légitimité d'une constitution - j'y reviendrai -, mais aussi sur le thème d'une " pluralité de loyautés » qui devient, portée à un certain degré, une menace. C'était là le thème de l'article de Schmitt auquel se réfère Böckenförde à plusieurs reprises, «Éthique de l'État et État pluraliste ", paru en 1930 : l'État démocratique-libéral était décrit comme pris en tension entre ces deux pôles, le pluralisme des opinions et des appartenances politiques, et l'unité d'une éthique qui devrait subordonner le pluralisme de la société civile au souci d'unité. Or cette unité se dissout sous Weimar, avec l'apparition de ce que Schmitt nomme, au début des années 1930, des "partis totaux » - le parti communiste, le parti national-socialiste, porteurs de "visions du monde " radicales et antagoniques. Böckenförde écrit de son côté, dans son essai critique Der deutsche Katholizismus im Jahre 1933 : « la République de Weimar [...] n'a pas eu, depuis le début, de défenseur sérieux et résolu de sa substance politique elle-même fondée sur un compromis politique et un compromis de vision du monde $»^{5}$. Les sociaux-démocrates la soutenaient mais en tant que transition et ouverture vers le socialisme (selon le mot de Bernstein que rappelle Böckenförde : «Republik, das ist nicht viel, Sozialismus ist das Ziel »); une réserve comparable se retrouvait chez une grande part des catholiques, si l'on substitue ici «christlicher Staat » à "Sozialismus ». Lorsqu'elle est distribuée entre des forces politiques et sociales dont les buts impliquent au mieux le dépassement au pire la destruction de la forme d'État en vigueur, la pluralité de loyautés finit par faire dépérir la démocratie elle-même. Mais là encore, si les diagnostics sont communs (les limites de la pluralisation des loyautés), Schmitt en venait à une conclusion que Böckenförde ne reprend pas à son compte telle quelle : la transformation nécessaire de l'État libéral en un État autoritaire, en une démocratie non-libérale. Catholique de gauche, social-démocrate, Böckenförde réoriente la question, dans les années 1960-1970, vers la construction nécessaire d'un État de droit social. Il écrit ainsi : « une des tâches centrales du nouvel ordre politique en Allemagne après 1945 [...] fut la reconstruction [Wiederherstellung] et l'aménagement de l'État de droit [...] : à la place de l'État de droit formel devait advenir un État de droit matériel, à la place de l'État de droit libéral un État de droit social $»^{6}$.

8 Le souci de combiner État de droit libéral et État social s'inspire bien d'un diagnostic rétrospectif des faiblesses de Weimar et, plus généralement, d'un libéralisme " pur ", qui refuserait toute intervention « matérielle » de l'État et limiterait l'action de celui-ci à une protection des libertés. 
9 L'État de droit social, à la différence de l'État de droit libéral stricto sensu, veille positivement à ce que la société ne soit pas trop fragmentée, à ce que des pans entiers de la population ne tombent pas dans une détresse sociale qui empêche de se vivre comme partie intégrante d'une société dite démocratique. Contrairement à bien des juristes libéraux et conservateurs, Böckenförde estime dans un article de 1974 que "l'intégration des droits sociaux dans l'édifice d'un État de droit démocratique » est tout à fait possible et souhaitable, qu'elle n'est pas contraire à la sauvegarde des libertés de l'État de droit libéral-bourgeois, mais constitue plutôt le développement de cette sauvegarde dans une situation sociale nouvelle.

10 Certes, cette réflexion sur l'unité sociale minimale requise comme précondition pour la survie d'une unité juridique, d'une constitution, a bien d'autres canaux et d'autres formes dans l'Allemagne d'après 1945. On la retrouve dans l'ordolibéralisme d'Eucken, en direction d'une action économique de l'État qui tient compte de la " fragilité » de la société - fragilité qui est apparue en pleine lumière avec la crise de 1929. L'État doit protéger la société de son éclatement, ce dont le marché, laissé à lui-même, n'est pas capable. On retrouve aussi cette réflexion sous une autre formulation dans les programmes du SPD. Mais articuler l'État de droit social au thème, repris de Schmitt, de l'homogénéité politique, est une opération originale de Böckenförde.

\section{Limites de la révision constitutionnelle}

11 La reconstruction d'un ordre constitutionnel après 1945 et à l'école de la faillite de Weimar a d'autres dimensions, plus proprement constitutionnelles.

12 Le cœur proprement constitutionnel de la question, c'est le problème des limites de la révision constitutionnelle, ou d'un éventuel «noyau » qui devrait, par principe, échapper sinon à la révision, du moins aux modalités ordinaires de la révision, parce qu'ils engagent une décision politique fondamentale - l'identité du régime, en quelque sorte.

13 C'était un des axes de la Théorie de la constitution de Schmitt (1928), et encore de Légalité et légitimité, avec sa fameuse question: une Constitution peut-elle "fournir une méthode légale pour l'abolition de sa propre légalité et [...] le moyen légitime de la destruction de sa propre légitimité »?

Dans son article de 1983 sur «Le développement historique et le changement de signification de la constitution", Böckenförde revient sur les discussions constitutionnelles qui ont eu lieu après 1945, et il observe que ces discussions se rattachaient à une problématique et à des positions datant des débats autour de la Constitution de la République de Weimar. Une section de l'article est consacrée, là encore, à la Constitution de Weimar comme compromis de classes (Klassenkompromiß). Un passage rassemble un certain nombre de problèmes: "elle [la Constitution de Weimar] est née après la fin d'une guerre et une révolution, comme modus vivendi (provisoire) entre des groupements politiques qui se faisaient face sur un mode antagonique, de "droite" et de "gauche", dont chacun suivait en fait des buts constitutionnels différents, et un "centre" [Mitte], soutien de l'État, i. e. engagé pour la République libérale-démocratique, qui devint assurément de plus en plus faible au cours du temps. La Constitution de Weimar ne contenait par conséquent pas de décision univoque [eindeutig] pour un ordre social déterminé, par exemple pour l'ordre 
(bourgeois) existant. Elle ne contenait qu'une série de garanties isolées, en partie hétérogènes les unes vis-à-vis des autres, pour l'ordre social et économique (art. 151-165), le compromis sur la socialisation et la propriété des articles 153-156 et avant tout la "neutralité" vis-à-vis de n'importe quelle modification constitutionnelle dans l'article 76, de telle façon que s'il existait une majorité des deux tiers, tous les contenus de la constitution pouvaient être remis en question $»^{7}$.

Cette ouverture de principe à une révision constitutionnelle radicale pose précisément, me semble-t-il, le problème de l'absence de fondements normatifs clairs de l'État. Böckenförde rappelle alors la position représentée par une partie des juristes de Weimar dans les années 1928-1932 - et il cite Carl Bilfinger, Heinrich Triepel et Carl Schmitt - selon laquelle "l'autorisation à apporter des modifications constitutionnelles selon l'article 76 ne s'étendait pas aux décisions fondamentales [Grundentscheidungen] de la Constitution». C'était là, ajoute Böckenförde, une « tentative de limiter l'ouverture de cette constitution qui résultait du compromis, de la limiter en faveur de l'inattaquabilité de ses principaux énoncés (démocratie, État de droit) pour faire front à la "révolution légale" prônée par le parti communiste allemand et le parti national-socialiste $»^{8}$.

En effet, d'un côté, Lénine avait indiqué la ligne à suivre aux partis communistes : « un révolutionnaire qui n'utilise pas tous les moyens, illégaux et légaux, est un piètre révolutionnaire ». Et Hitler avait expliqué lui-même en 1930 la stratégie de révolution légale, après l'échec de ses premiers putschs: "il ne faut pas chercher à renverser l'État, mais y pénétrer par les voies légales, pour s'en rendre maître et le transformer : battre la démocratie avec ses propres armes et parvenir à la dictature grâce au suffrage universel $»^{9}$.

Schmitt avait donc quelque motif, en 1932, de s'inquiéter des limites du principe libéral des « chances égales » d'accès au pouvoir qui devraient être garanties à tout parti, quels qu'en soient les buts politiques. Dans la Théorie de la constitution comme dans Légalité et légitimité (1932), il pointait la tension, voire la contradiction entre deux pôles ou deux parties principales de la Constitution de Weimar: d'un côté, un système formel de limitation du pouvoir et de libre concurrence des partis, indépendamment de toute référence à des valeurs; de l'autre, une seconde partie qui renvoie à des valeurs, à travers la notion de «droits fondamentaux » (Grundrechte).

Cette seconde partie était largement l'œuvre de Friedrich Naumann, qui, note Schmitt, dut s'opposer au positivisme dominant pour avancer cette contribution. Or Schmitt écrit ceci dans Legalität und Legitimität : «Lorsque F. Naumann, en butte aux sarcasmes d'un positivisme inconscient, rédigea son projet sur les droits fondamentaux, quelque vague et imprécise que fût son idée, elle avait plus d'affinités avec le caractère d'une constitution allemande que la constitution d'un système majoritaire qui fait abstraction des valeurs $»^{10}$. Schmitt souligne ensuite que cette partie sur les droits du citoyen « jette les bases d'un ordre social » et que ses principes devraient être développés.

19 La Constitution doit donc assumer sa dimension normative " réelle », pourrait-on dire : une Constitution n'est pas seulement un système de normes dérivées logiquement d'une norme fondamentale posée par le législateur, comme le veut Kelsen; c'est un instrument de définition de soi, un acte par lequel un peuple s'institue politiquement comme peuple, définit les bases de l'ordre social tel qu'il le conçoit. L'ambiguïté de Legalität und Legitimität tient à ce que Schmitt dit que ce serait là la vraie constitution 
allemande, et l'acte par lequel le peuple se donne ses normes politiques est décrit comme un acte politique, donc polémique.

Dans son analyse des deux pôles ou des "grandes parties " de la Constitution de Weimar, la critique du formalisme de la «libre concurrence des partis", de la neutralité et de l'objectivité de l'État libéral qui devrait laisser ce jeu s'opérer sans considérer les contenus politiques a également une dimension nationaliste : Naumann cherchait cette « substance » dans les droits fondamentaux, mais c'est aussi ou d'abord, pour Schmitt, la dimension de la Constitution qui renvoie au peuple allemand dans sa particularité.

Dans la Théorie de la constitution, de 1928, Schmitt parlait plutôt favorablement du "pathos politique» de Naumann et de sa volonté, avec la déclaration des droits fondamentaux, de donner à la Constitution allemande l'équivalent d'une « déclaration solennelle » qui serait, écrit Schmitt, « l'expression naturelle de la conscience qu'ont les acteurs d'imprimer une direction déterminée à leur propre destin politique ». Mais Schmitt rapprochait alors cette forme de déclaration des Déclarations américaine de 1776, française de 1789, allemande de 1848 et soviétique de 1918. Il ne valorisait aucunement par là la dimension " universaliste » de telles déclarations des droits de l'homme «à la 1789 » par exemple, mais bien plutôt la résolution d'un peuple particulier à se donner une orientation politique, et à polariser ainsi une éventuelle hostilité contre lui : de tels actes politiques "présupposent qu'un peuple prend sur lui par sa déclaration le risque d'un regroupement politique radicalement nouveau, c'est-à-dire un regroupement entre ami et ennemi, et est résolu à défendre les nouveaux principes de son État en luttant même contre un puissant ennemi extérieur $»^{11}$.

D'un côté, on peut entendre là des échos d'un pathos de la résolution qu'on trouvait, un an plus tôt, chez Heidegger, et qui, selon Karl Löwith, a participé, avec le pathos de la décision, de ces affinités politiques qui ont compté dans le ralliement ultérieur de Schmitt et de Heidegger au III ${ }^{e}$ Reich.

Mais d'un autre côté, Schmitt désignait ici l'Assemblée constituante de 1789, la réflexion de Sieyès sur le pouvoir constituant comme la véritable novation d'une "décision globale libre, prise par des hommes, sur le genre et la forme d'une décision politique $»^{12}$, et c'est cet exemple qui lui fait dire que la constitution ne repose pas véritablement sur une "norme ", mais sur une décision et une volonté émanant d'un " être », i. e. qu'une constitution a un caractère « existentiel $»^{13}$. On peut dire à ce titre que la problématique schmittienne est en décalage par rapport à celle des fondements normatifs de l'État constitutionnel: elle vise moins les fondements normatifs de l'État constitutionnel que ses fondements politiques qui, précisément, ne relèveraient jamais, en dernière instance, de normes. Il s'agit là d'une thèse frappante, mais certainement hautement contestable.

Néanmoins, la distinction de la Théorie de la constitution entre un pôle formel et un pôle substantiel de la Constitution, entre Verfassung et Verfassungsgesetz avait une force propre. Articulée à la question des limites de la révision constitutionnelle, elle peut être mise au service d'une défense des libertés fondamentales comme socle "indérogeable » d'une Constitution démocratique-libérale ou républicaine, noyau de constitutionnalité qui échappe par principe à la révision, socle de légitimité qui engage l'identité politique du régime ; ici, la «neutralité en valeur ", le formalisme de règles valables quelles que soient les forces politiques qui s'emparent du gouvernement ne sont plus de mise. Est-ce à dire qu'on limite constitutionnellement le pluralisme démocratique? Si l'on veut, 
mais on le limite pour sauvegarder la légitimité démocratique contre un usage dévoyé de la légalité, de la légalité de la "concurrence politique ouverte» à chances égales, y compris pour des forces antidémocratiques.

Comme on sait, c'est bien la voie choisie par la République fédérale d'Allemagne après 1945 : la Loi fondamentale de 1949 prévoit à son article 21 alinéa 2 la possibilité d'une interdiction comme «inconstitutionnels » des partis dont le projet ou le programme prévoit la destruction de la forme de l'État démocratique, ouvert au pluripartisme et acquis au principe d'une possible révocation des détenteurs du pouvoir par voie électorale. Une telle interdiction fut appliquée en 1953 pour le parti néo-nazi Sozialistische Reichspartei, et pour le parti communiste (KPD) en 1956 - interdiction levée, dans ce deuxième cas, en 1968.

On comprend que Carl Schmitt ait pu juger que tout cela confirmait après-coup la justesse de ses analyses concernant la période de Weimar, même s'il le fait en 1949 avec cette exaspérante auto-satisfaction qui caractérise souvent ses écrits d'après-guerre : "lorsque je lis la Loi fondamentale, j'éprouve l'amusement d'un vieillard omniscient $»^{14}$. La Loi fondamentale de la République de Bonn correspondrait à ce Verfassungsgesetz qui constitue, selon une expression que Schmitt empruntait à Hauriou, une "supralégalité", et ferait de la Constitution, pour citer cette fois Böckenförde, un «ordre juridique et un fondement en valeur de la communauté politique» («rechtliche Lebensordnung und Wertgrundlage des politischen Gemeinwesens »).

27 Là encore, l'ordre constitutionnel d'après 1945 puisait dans les débats de Weimar et, estime Böckenförde, retrouvait deux tentatives éminentes pour surmonter le positivisme d'avant-guerre : d'un côté, la « doctrine de l'intégration » de Rudolf Smend, pour qui la Constitution a une fonction d'intégration dynamique de contenus normatifs en évolution. La Constitution "capte" et donne une forme unitaire à des valeurs culturelles. À quoi Böckenförde oppose la position de Schmitt : la constitution comme décision, et comme "point d'unité (fondé statiquement) qui précède [vorausliegt] les "normations" des lois constitutionnelles [verfassungsgesetzlich] mais qui peut être juridiquement accompli dans ces dernières $»^{15}$. Böckenförde renvoie ici au $\S 3$ de la Théorie de la constitution de Schmitt, mais il suggère que ce n'est pas la position de Schmitt qui a prévalu après 1945, mais bien la compréhension de la Constitution référée à un "ordre de valeurs » lui-même pensé avec le soutien de la philosophie des valeurs - de Scheler et de Hartmann, sorte de kantisme dynamisé par la Lebensphilosophie.

Or Böckenförde estime que c'est là une fondation normative insuffisante : « le problème de la compréhension en termes de valeurs [...] réside en ceci qu'il n'existe pas une connaissance rationnellement contrôlable des "valeurs" et d'un "ordre de valeurs" ${ }^{16}$, pas plus qu'il n'existe de moyen de trancher «scientifiquement» ou gnoséologiquement lorsqu'on est confronté à la prétention à la validité de plusieurs systèmes de valeur. Böckenförde fait alors sienne une certaine critique schmittienne de la philosophie des valeurs, qui ne nous dispenserait nullement, en fait, d'une " décision » quant à l'échelle et à la priorité de telle ou telle valeur. Comment établir l'ordre de priorité des valeurs présentes dans la Constitution de Bonn - la liberté, l'égalité, la justice, la sécurité, la réalisation de soi, la solidarité, la protection de la vie? Böckenförde estime que la justification fondamentale, métapositive (metapositive Fundamentalbegründung), espérée et attendue d'une philosophie des valeurs, n'a pas été 
offerte, ce qui a donné une très grande importance à l'application et à l'interprétation de la constitution - notamment, peut-on penser même si Böckenförde ne le dit pas ici, à son interprétation par la Cour constitutionnelle de Karlsruhe. Est-ce que, dès lors, la « décision » sur la communauté politique, à travers une décision sur la priorité de telle ou telle valeur/principe, n'était pas ainsi transférée vers les juges constitutionnels? N'estil pas alors problématique que la "décision» sur les fondements politiques de la communauté politique du peuple soit confiée, non à une instance politique (le peuple ou son représentant), mais à une instance juridique?

On est alors renvoyé au débat entre Schmitt et Kelsen : qui doit être le gardien de la constitution? Mais Böckenförde clôt son article sur le constat d'une insuffisance de l'appel aux valeurs, sans préciser les moyens d'y remédier.

Böckenförde s'accorde donc bien partiellement avec Schmitt sur la critique d'une tentative de fondation de l'État par l'invocation de «valeurs ", d'une «philosophie des valeurs".

31 Ce qui est frappant, cependant, c'est le fait que, si le diagnostic sur l'insuffisance d'une philosophie des valeurs pour assurer l'homogénéité politique est en partie schmittien, la réponse normative de Böckenförde s'écarte de Schmitt et est tout à fait... hégélienne. L'article fameux sur la naissance de l'État comme processus de sécularisation renvoie à l'essai de Schmitt, Die Tyrannei der Werte, le dernier paragraphe du texte, qui contient le fameux « théorème » (« l'État libéral moderne sécularisé vit de présupposés qu'il n'est pas capable de garantir lui-même »), en appelle en effet à la philosophie du droit de Hegel, et à son prolongement par le juriste Lorenz von Stein. Ce sont Hegel et Stein qui permettent de penser l'intégration de la liberté et de la pluralité qu'elle implique à un ordre, la réconciliation du principe "organique » mis en avant par Aristote avec le principe « individualiste » qui est le principe des Temps Modernes pour Hegel, affirmé par la Réforme et la Révolution, même s'il a été d'abord porté, au plan de l'Histoire de l'Esprit, par le christianisme. L'État libéral n'est pas fondé sur un pur renvoi aux valorisations subjectives et relatives, dans leur pluralité infinie (ce qui était au fond la thèse de Kelsen dans son essai sur la démocratie de 1922 : la démocratie, au fond, comme relativisme); l'État démocratique moderne est le produit d'une sécularisation qui a fait des principes chrétiens de valorisation de la subjectivité libre et de l'égalité de chacun «en Dieu» les principes d'un nouveau monde politique et juridique, des principes « fondateurs » de l'ordre politique, précisément.

Un écart profond sépare Böckenförde de Schmitt à cet égard, comme l'expriment les dernières phrases de l'article sur le concept de politique de Schmitt comme clé de son œuvre constitutionnelle : « la question de la signification de la liberté - de la liberté individuelle et de la liberté politique - pour l'unité et l'ordre étatiques. Est-elle [cette signification] vue et déterminée de façon adéquate dans l'œuvre de droit public de Carl Schmitt, ou bien de façon restrictive, notamment en ce qui concerne la formation et le déploiement de soi de l'homogénéité relative, les liens réciproques au sein d'un peuple, comme fondement de l'unité et de la capacité d'action étatiques?» ${ }^{17}$. Böckenförde termine son article sur ce point d'interrogation, et ce silence vaut, bien sûr, réponse réponse négative.

Dans son essai de 1961, Der deutsche Katholizismus im Jahre 1933, Böckenförde s'écarte résolument de l'antimodernisme et de l'antilibéralisme dont il estime qu'ils sont en partie responsables des trop grands accommodements de pans entiers du catholicisme allemand avec Hitler. Contre une vision organiciste de la société, Böckenförde en 
appelle, là encore, à la vision hégélienne, qui a su concilier deux pôles : d'un côté, les principes fondamentaux de la modernité politique, la reconnaissance de la subjectivité libre affirmée par la Réforme protestante et la Révolution française, et, d'un autre côté, un souci d'ordre, d'organisation différenciée d'un corps social. Les normes éthiques qui sous-tendent l'ordre démocratique-«libéral », donc les fondements prépolitiques de l'État démocratique (selon la reformulation de Habermas), sont pensées par Böckenförde, à l'instar de Hegel, comme des normes issues essentiellement de la sécularisation du christianisme et de sa valorisation de l'intégrité et de l'unicité de la personne. Et c'est encore avec Hegel que Böckenförde entend défendre la légitimité de l'Entzweiung, de la dualité ou division entre société civile et État, qu'il tient pour une condition de la liberté individuelle et non pour une simple expression de la domination bourgeoise et de sa scission abstraite entre privé et public, comme le veut Marx.

\section{Capitant : État fort mais fondements libéraux de l'État constitutionnel républicain}

34 Si l'on en vient à la comparaison franco-allemande, il faut remarquer que les positions de Schmitt sur le problème des présupposés politiques de la constitution ont trouvé en France un écho, une reprise et un complément normatif-critique largement comparables à la lecture critique opérée par Böckenförde, chez René Capitant.

Il y a d'ailleurs là un certain chassé-croisé : la réflexion de Schmitt dans les années vingt s'inspire pour une bonne part du courant institutionnaliste français, Hauriou en particulier ; après la Seconde Guerre mondiale, la pensée constitutionnelle de Schmitt a un effet souterrain sur les travaux constitutionnels français qui déboucheront sur la $V^{e}$ République, via René Capitant.

Capitant avait en effet suivi attentivement les débats constitutionnels allemands sous Weimar. Or dans son article "Le rôle politique du Président du Reich», paru en mars 1932 dans la revue Politique, le futur collaborateur de Léon Blum et du général de Gaulle estime que les arguments de Schmitt en faveur du Président du Reich comme "gardien de la constitution », tout comme ses réflexions sur la possibilité de suspendre certains aspects de la Constitution pour en sauver le principe, sont convaincants.

Ils sont plus convaincants, aux yeux de Capitant, dans la perspective d'un sauvetage de la République, que le recours à un contrôle permanent de constitutionnalité prôné en réponse par Hans Kelsen. Les arguments de Kelsen, qui contestent qu'un Président élu soit plus «neutre » et plus indépendant des partis qu'une Cour constitutionnelle et que des magistrats dont l'ethos professionnel implique une telle neutralité, sont sans doute justes dans une situation normale. Mais le débat porte précisément sur une situation où " garder » la Constitution, la " préserver » signifie autre chose qu'un simple contrôle de constitutionnalité.

Dans les circonstances de quasi-guerre civile que vit l'Allemagne, estime Capitant, "sauvegarder la République, cela ne signifie pas exercer de contrôle de constitutionnalité sur chacune des lois votées, et la réponse qu'adressait récemment M. Hans Kelsen à M. Carl Schmitt pour défendre [...] l'institution d'une Cour constitutionnelle, est évidemment sans portée. Il ne s'agit pas de confier au Président l'exercice d'une fonction juridictionnelle, mais bien plutôt de lui demander de parer au coup d'État et de sauver en Allemagne les institutions démocratiques et parlementaires. 
Pour cela, la Constitution lui donne des armes $»^{18}$, ajoute Capitant, en se référant à l'article 48, tant commenté par Schmitt. Capitant suit l'argumentation de Schmitt, sur le fait que des «partis anticonstitutionnels » faussent le jeu démocratique. Ainsi, ajoute Capitant, " on peut se demander si, en certaines circonstances, le véritable loyalisme n'obligerait pas le Président à violer la lettre de la Constitution pour en sauver le principe ». Mais Capitant précise aussitôt, beaucoup plus explicitement que ne le faisait Schmitt dans Legalität und Legitimität, ce qu'il a en vue : "Que le Reichstag offre à Hitler la majorité qu'il lui refuse encore, que le pays se prononce dans le même sens, la légalité constitutionnelle impose alors au Président d'appeler le chef national-socialiste au poste de Chancelier, mais la vraie fidélité à la Constitution l'admet-elle ? ${ }^{19}$.

Ici, on pourrait dire que la question est bien, en un sens, celle des fondements normatifs de l'État constitutionnel, en tant que ces fondements peuvent être vus comme méta-positifs, comme engageant une «légitimité » qui peut, le cas échéant, conduire à s'opposer à une fausse légalité, ou à un usage hypocrite de la légalité. Seulement ces "normes ", et c'est tout le problème, ne sont présentes en l'occurrence que dans un certain « esprit » de la constitution démocratique dont le Président serait le garant. Capitant, en tant que gaulliste, assuma cette dimension "personnaliste", comme disait parfois Schmitt, d'un esprit de la constitution, ou d'une légitimité démocratique qui résiderait parfois dans un homme plutôt que dans des normes ou dans la lettre des lois, de même qu'il entendait défendre ce qu'il appelait « l'État fort de la démocratie " contre "l'État faible des libéraux». Le renforcement du pouvoir présidentiel dans la Constitution de la $\mathrm{V}^{\mathrm{e}}$ République. - même si celle-ci n'est pas, bien sûr, l'œuvre de Capitant, mais une création collective - participe de cet esprit. Carl Schmitt était persuadé de cette continuité, et il écrit à Armin Mohler, le 18 mai 1961 : «L'essai de Capitant de 1932 sur le gardien de la Constitution [...] est l'élément qui fait lien [Verbindungsglied]entre mon Hüter der Verfassung et la Constitution de Gaulle ${ }^{20}$. Il pense en particulier à l'article 16 de la Constitution de 1958, sur les pouvoirs exceptionnels.

Néanmoins, chez Capitant comme chez Böckenförde, un certain souci de l'identité politique incarnée dans la décision et dans un État fort ne conduisait nullement à récuser en bloc les normes libérales et universalistes, comme le fit de plus en plus Schmitt, surtout, bien sûr, dans la période nazie. Dans certaines lettres et dédicaces à Schmitt, Capitant marque qu'il sait bien que l'écart entre eux est profond, sur ce point de l'adhésion à des valeurs de liberté.

Dans ses Cours de droit (public) de 1952-53, René Capitant met en avant, à titre de fondement normatif de la démocratie, un "postulat spiritualiste », celui de la «liberté individuelle ». Rousseau, qui est pour Capitant le fondateur de la démocratie moderne chez qui il faut chercher les éléments de définition et de compréhension de la démocratie, fait commencer le Contrat social par les mots « l'homme naît libre » (en fait : «l'homme est né libre»), et la lecture du dernier chapitre du Contrat social «laisse apparaître en toute clarté les fondements spiritualistes de la doctrine de Rousseau - un spiritualisme nourri d'évangélisme chrétien $»^{21}$.

Plus loin, Capitant souligne que si l'on prétend fonder l'État, « non plus sur l'autonomie de la personne humaine, mais sur un principe métaphysique imposant à l'individu des obligations indépendantes de son consentement", sur "l'obligation unilatérale et inconditionnelle [des] sujets de se soumettre à une idéologie que [l'État] incarne et que les chefs interprètent souverainement», on a affaire alors au "contraire de la 
démocratie ", i. e. à des " dictatures totalitaires $"^{22}$. On est là aux antipodes des textes de Schmitt au moins d'après 1933, sur le Führer comme source de tout droit, «loi incarnée ", ou encore du livre de Schmitt de 1938 sur le Léviathan, que Capitant avait lu, où Schmitt déplorait la «faille » introduite entre État et société, le principe de la liberté intérieure, reconnu par Hobbes et élargi en liberté de conscience par «le premier juif libéral », Spinoza.

Après avoir essayé de suivre quelques transformations et dépassements des concepts schmittiens chez Böckenförde et plus succinctement chez Capitant, je voudrais avancer une appréciation, non pas de la valeur propre de ces constructions, mais de l'actualité des résultats et des thèmes que j'ai évoqués. Cette réflexion s'est construite en ayant en vue ou à l'esprit un État démocratique précaire, confronté à des forces radicalement antilibérales, comme la République de Weimar, dont la République de Bonn devait conjurer le spectre; la $\mathrm{V}^{\mathrm{e}}$ République également est née sur fond de chute d'une République. Dans quelle mesure les États démocratiques contemporains sont-ils menacés par des « partis totaux » qui bénéficieraient du soutien d'une part importante de la population et joueraient un double jeu avec la légalité démocratique? Dans son livre Les Derniers jours de Weimar, Olivier Beaud se demandait si on ne devait pas déplacer vers l'extrême droite actuelle les arguments de Schmitt en 1932, pour recommander, par exemple, l'interdiction du Front national. Mais il répondait négativement, non pas au nom d'une neutralité en valeur ou sous l'effet d'une banalisation de ce parti dont on entend aujourd'hui dire par d'innombrables observateurs complaisants, à la faveur de son « rajeunissement » de façade, qu'il est un parti démocratique comme les autres, mais parce qu'un État démocratique fort peut et doit maintenir le jeu aussi ouvert que possible. Le thème de la "révolution légale » ne fait plus recette, peut-être parce que le thème de la révolution tout court a perdu de son aura dans le monde occidental, mais aussi parce que la perspective de la dictature n'est plus mise en avant, même par les partis d'extrême droite ou d'extrême gauche. Néanmoins, s'il y avait une probabilité réelle pour que le Front national accède au pouvoir, la question de la Grundentscheidung se poserait bien, c'est-à-dire la question de savoir si la démocratie doit être identifiée au principe majoritaire, ou si elle engage des normes fondamentales (égalité de droits indépendamment des origines, droits de l'homme) dont on peut penser que le Front national, au-delà de l'interrogation sur le point de savoir s'il «fermerait la porte derrière lui » en cas de victoire, leur porterait nécessairement atteinte.

Concernant l'homogénéité, le problème actuel me semble être que l'État de droit social, défendu à juste titre par Böckenförde, tend à se déliter, ou plutôt à être activement démantelé. Or les forces politiques-gouvernementales qui réalisent activement cette destruction des conditions d'une homogénéité sociale minimale ont tendance à mobiliser dans le même temps le peuple-électeur autour d'une autre homogénéité, l'unité ou l'identité du peuple-national qui se définit toujours bien "polémiquement ". On régresse alors du Böckenförde de l'État de droit social au Schmitt de 1926 et de la démocratie xénophobe. Le rapprochement idéologique en cours entre droite de gouvernement et extrême droite fait bien ressurgir le spectre d'une "démocratie xénophobe », pensant "l'homogénéité " sur un mode "identitaire » plus que social. L'axiome böckenfördien selon lequel l'État libéral sécularisé vit de présupposés normatifs qu'il n'est pas en mesure de garantir risque alors d'être dévié et de servir non plus à réconcilier intimement les forces chrétiennes et les forces démocratiques autour des principes fondamentaux de liberté et d'égalité des personnes, mais à construire 
" polémiquement» une "identité » chrétienne des États européens contre la part de leurs propres populations qui est "historiquement» et "généalogiquement» nonchrétienne - en particulier : musulmane.

\section{NOTES}

1. E. W. BÖCKENFÖRDE, "Entstehung und Wandel des Rechtsstaatsbegriffs ", repris dans Recht, Staat, Freiheit, Francfort-sur-le-Main, Suhrkamp, 1991/édition augmentée 2006, p. 169 note 89 : «Was [den] Untergang [der Weimarer Republik] herbeiführte, war nicht ein Mangel an rechtsstaatlicher Freiheit, sondern der Mangel an politischer Homogenität und demokratischer Loyalität bei den verschiedenen Gruppen und dem Volke selbst. »

2. Carl schmiтt, Parlementarisme et démocratie, trad. fr. J.-L. Schlegel, Paris, éditions du Seuil, 1988, p. 107.

3. Ibid., p. 106.

4. Ibid., p. 111.

5. E. W. BÖCKENFÖRDE, Der deutsche Katholizismus im Jahre 1933. Kirche und demokratisches Ethos, Fribourg/Bâle/Vienne, Herder, 1988, p. 63. «Die Weimarer Republik hatte [...] von Anfang an keine ernsthaften und entschlossenen Verteidiger ihrer auf einem politischen und weltanschaulichen Kompromiß beruhenden politischen Substanz. »

6. E. W. BÖCKENFÖRDE, « Entstehung und Wandel des Rechtsstaatsbegriffs », in : Recht, Staat, Freiheit (note 1), p. 143. «Eine der zentralen Aufgaben der politischen Neuordnung in Deutschland nach 1945 [...] war die Wiederherstellung und Ausgestaltung des Rechtsstaats. [...] an die Stelle des formalen Rechtsstaates sollte nun der materielle Rechtsstaat, an die Stelle des liberalen der soziale Rechtsstaat treten. »

7. E. W. BÖCKENFÖRDE, « Geschichtliche Entwicklung und Bedeutungswandel der Verfassung », in : Staat, Verfassung, Demokratie, Francfort-sur-le-Main, Suhrkamp, 1991, p. 46. «[Die Weimarer Verfassung] war nach Kriegsende und Revolution entstanden als (vorläufiger) modus vivendi zwischen den sich antagonistisch gegenüberstehenden politischen Gruppierungen von 'rechts' und 'links', die eigentlich je andere Verfassungsziele verfolgten, und einer staatstragenden, d.h. für die demokratisch-liberale Republik engagierten 'Mitte', die freilich im Laufe der Zeit immer schwächer wurde. Die Weimarer Verfassung enthielt infolgedessen keine eindeutige Entscheidung für eine bestimmte bzw. die bestehende (bürgerliche) Gesellschaftsordnung, sondern eine Reihe untereinander zum Teil heterogener Einzelgarantien zur Wirtschafts- und Sozialordnung (Art. 151-165), den Eigentums- und Sozialisierungskompromiß der Art. 153-156 und vor allem die 'Neutralität' gegenüber jedweder Verfassungsänderung in Art. 76, so daß bei Vorhandensein einer Zweidrittelmehrheit alle Inhalte der Verfassung zur Disposition standen. »

8. Ibid., p. 47. « Die von einem Teil der Weimarer Staatsrechtslehre (Carl Bilfinger, Carl Schmitt, Heinrich Triepel u.a.) in den Jahren 1928-32 vertretene Ansicht, die Befugnis zu Verfassungsänderungen nach Art. 76 erstrecke sich nicht auf die Grundentscheidungen der Verfassung, war der Versuch, gegenüber der herannahenden 'legalen Revolution' von seiten der KPD oder NSDAP die aus dem Kompromiß resultierende Offenheit der Verfassung zugunsten der Unangreifbarkeit ihrer Kernaussagen (Demokratie, Rechtsstaat) zu begrenzen. »

9. Cité par David cumin, Carl Schmitt, Paris, éditions du Cerf, 2005, p. 117. 
10. Carl schmitт, Legalität und Legitimität, Berlin, Duncker \& Humblot, 1932, rééd. 1993, p. 91 ; trad. fr., Légalité et légitimité, trad. et introduction de W. Gueydan de Roussel, LGDJ, 1936, p. 101. Certains mots sont omis dans la traduction de Gueydan de Roussel, on pourrait traduire plus littéralement ce passage ainsi : «mais ce qu'il y avait de vague et de flou dans l'intention de Friedrich Naumann, lorsque, raillé par les rires d'un aveugle positivisme d'avant-guerre, il rédigea son projet sur les droits fondamentaux, avait cependant plus de rapport avec l'essence d'une constitution allemande que la neutralité en valeur d'un système majoritaire conçu sur un mode fonctionnaliste ".

11. Carl sснмітт, Théorie de la Constitution, trad. fr., Paris, PUF (« Léviathan »), 1993, p. 299.

12. Ibid., p. 213.

13. Ibid., p. 212.

14. "Bei der Lektüre des Bonner Grundgesetzes befällt mich die Heiterkeit eines allwissenden Greises. » Carl schмiтT, Glossarium. Aufzeichnungen der Jahre 1947-1951, 20.7.1949, Berlin, Duncker \& Humblot, 1991, p. 259.

15. E.W. BÖCKENFÖRDE, «Geschichtliche Entwicklung und Bedeutungswandel der Verfassung» (note 7), p. 49-50. «[Die Verfassung als Entscheidung] sollte den (statischgründenden) politischen Einheitspunkt darstellen, der als solcher verfassungsgesetzlichen Normierungen vorausliegt, in ihnen aber juristisch vollziehbar wird. »

16. Ibid., p. 51. «Das juristische Problem des werthaften Verfassungsverständnisses [...] liegt darin, daß es eine rational kontrollierbare Erkenntnis von 'Werten' und einer 'Wertordnung' nicht gibt [...]. »

17. E. W. BÖCKENFÖRDE, « Der Begriff des Politischen als Schlüssel zum staatsrechtlichen Werk Carl Schmitts ", repris in Recht, Staat, Freiheit (note 1), p. 366. «Die Frage nach der Bedeutung der Freiheit - der individuellen und der politischen Freiheit - für die staatliche Einheit und staatliche Ordnung. Ist sie im staatsrechtlichen Werk Carl Schmitts zutreffend oder nur verkürzt gesehen und bestimmt, nicht zuletzt im Hinblick auf die Bildung und das Sich-Forttragen der relativen Homogenität, des Miteinanderverbundenseins in einem Volk als Grundlage der staatlichen Einheit und Handlungsfähigkeit?»

18. René CAPITANT, «Le rôle politique du Président du Reich », Politique, mars 1932, repris dans Écrits d'entre-deux-guerres, Paris, Éd. Panthéon-Assas, 2004, ici p. 402.

19. Ibid.

20. Carl schмiтt, lettre à Armin Mohler du 18 mai 1961, Carl Schmitt - Briefwechsel mit einem seiner Schüler, Berlin, Akademie Verlag, 1995, p. 306.

21. René CAPITANT, « Cours de droit de 1952-53», Titre second : «Les principes fondamentaux de la démocratie ", in : Écrits constitutionnels, Paris, éd. du CNRS, 1982, p. 152.

22. Ibid., p. 172.

\section{RÉSUMÉS}

Tant René Capitant qu'Ernst-Wolfgang Böckenförde ont fait un usage inventif et critique de concepts schmittiens, qu'ils transforment et dépassent dans leur œuvre. Sur fond d'interprétation de la crise de Weimar, il s'agit notamment de la notion d'homogénéité politique, des limites de la révision constitutionnelle et du rapport entre Constitution et forme politique. Böckenförde les réoriente dans la perspective d'une "consolidation » de l'État démocratique 
comme «État de droit social ». La question des fondements normatifs de l'État suppose, quant à elle, de s'écarter de Schmitt au plan axiologique.

Sowohl René Capitant als auch Ernst-Wolfgang Böckenförde hatten einen schöpferischen und kritischen Umgang mit Begriffen Carl Schmitts, über die sie in ihrem Werk reflektieren und hinausgehen. Auf dem Hintergrund der Interpretation der Weimarer Krise geht es unter anderem um den Begriff der politischen Homogenität, um die Grenzen der Verfassungsrevision und um das Verhältnis zwischen Verfassung als Text und Verfassung als politischer Form. Böckenförde interpretiert sie neu im Sinne einer Stärkung des demokratischen Staates als sozialen Rechtsstaates. Die Frage nach den normativen Grundlagen des Staates setzt ihrerseits eine axiologische Abgrenzung von Schmitt voraus.

\section{AUTEUR}

\section{JEAN-CLAUDE MONOD}

Chargé de recherche au CNRS, UMR 8547 (CNRS/ENS), Archives Husserl de Paris 